\title{
Design and Analysis of a TEM Mode Rectangular Coaxial Waveguide for Mobile 5G Millimeter Wave Antenna Module Applications
}

\author{
Eunyoung Park · Sangkil Kim*
}

\begin{abstract}
In this paper, design and analysis of a transverse electromagnetic (TEM) mode rectangular coaxial waveguide for a mobile $5 \mathrm{G}$ millimeter wave (mmWave) antenna module are presented. General structures of $5 \mathrm{G}$ radio frequency (RF) module for mobile sub-6 $\mathrm{GHz}$ and $\mathrm{mmWave}$ applications are also discussed in this paper. Thorough analysis of transmission line and waveguide structures at mmWave frequency band is essential and fundamental information to design highly integrated RF front-end modules. Theoretical design equations of the waveguide, such as impedance, loss, and radiation are presented, and the equations are verified by a full wave 3D FEM electromagnetic simulator. Impedance value of the rectangular waveguide structure was calculated using the conformal mapping method. Theoretical operation frequency bandwidth and design guide are also presented. The characteristics and analysis of the rectangular coaxial waveguide structure presented in this paper is easily scalable to other frequency bands. The proposed design equations are also applicable to various planar layer-by-layer integrated circuit (IC) or module manufacturing process.
\end{abstract}

Key Words: Millimeter-Wave, mmWave Module, RF Front-End, TEM Mode, Waveguide, 5G Communication System.

\section{INTRODUCTION}

In April 2019, a commercial sub-6 GHz band mobile 5G communication network service was launched in South Korea for the first time. The $5 \mathrm{G}$ communication features an ultra-fast data rate up to several Gbps and ultra-low latency of several ms. Various unprecedented user experiences and technologies, such as augmented reality (AR), smart factory/hospital, cloud computing, and self-driving cars, are available due to the $5 \mathrm{G} \mathrm{com-}$ munication network $[1,2]$. The next technical challenge is to implement millimeter wave (mmWave) $5 \mathrm{G}$ technology operating at $28 \mathrm{GHz}$ or $39 \mathrm{GHz}$. This technology is able to improve data rates dramatically by fully utilizing its broad bandwidth of 800-1,000 MHz. The wavelength at the mmWave 5G frequency band is less than $10 \mathrm{~mm}$, which is a comparable size to radio frequency front-end (RFFE) module chips. For this reason, the antennas for mmWave $5 \mathrm{G}$ communication could be implemented in the package (antenna-in-package [AiP]) [3]. The distance between the radio frequency integrated circuit (RFIC) and the antenna could be minimized, and the mmWave signals could be tightly confined within the waveguide structures.

The $5 \mathrm{G}$ mmWave module was designed using system-inpackage (SiP) technology $[4,5]$. Many functional chips and

Manuscript received January 9, 2020 ; Revised March 24, 2020 ; Accepted March 30, 2020. (ID No. 20200109-006J)

Department of Electronics Engineering, Pusan National University, Busan, Korea.

"Corresponding Author: Sangkil Kim (e-mail: ksangkil3@pusan.ac.kr)

This is an Open-Access article distributed under the terms of the Creative Commons Attribution Non-Commercial License (http://creativecommons.org/licenses/by-nc/4.0) which permits unrestricted non-commercial use, distribution, and reproduction in any medium, provided the original work is properly cited.

(c) Copyright The Korean Institute of Electromagnetic Engineering and Science. All Rights Reserved. 
passive components, such as power management integrated circuit (PMIC), RFIC, antenna, and SMDs, were integrated in a single package laminate, and the module was connected through a flexible RF cable. The RF cable is a critical component, as DC power, analog/digital control, and intermediate frequency (IF) signals are carried by it. Therefore, it is essential for the $5 \mathrm{G}$ mmWave module to design and analyze the RF cable thoroughly. This paper discusses the design requirements of the RF cable and presents a proper waveguide structure with thorough theoretical analysis.

\section{5G MMWAVE MODULE FOR MOBILE DeVICES}

A high-frequency module is a key component of the RFFE chain. Usually, low noise amplifiers (LNAs), power amplifiers (PAs), filters, duplexers, and antenna switches are integrated into a single chip using the $\mathrm{SiP}$ technology. It is an essential part of mobile wireless communication systems because it is able to integrate many IC chips made through various processes (CMOS, GaAs, or SiGe) into a single module chip. The general structure of an $\mathrm{RF}$ module for $5 \mathrm{G}$ applications is shown in Fig. 1.

The most widely used commercial sub- $6 \mathrm{GHz}$ band $\mathrm{RF}$ modules for 4G-LTE and $5 \mathrm{G}$ communication consist of $\mathrm{Rx} / \mathrm{Tx}$ amplifiers (LNAs and PAs), an antenna tuning system, and multiplexers (Fig. 1). The antennas are not integrated within the module because they are much larger than the module chip at the sub- $6 \mathrm{GHz}$ frequency band $(3.6 \mathrm{GHz})$. However, the operating frequency bands for $5 \mathrm{G}$ mmWave RFFE module are 28 $\mathrm{GHz}$ and $39 \mathrm{GHz}$, and their half-wavelength $\left(\lambda_{g} / 2\right)$ is less than $5 \mathrm{~mm}$ in the free space. It is feasible to integrate antennas and RFICs in a single package (module chip) due to an antenna size comparable to the RFICs.

The world's first commercially available $5 \mathrm{G}$ mmWave module chip for mobile devices also has a structure similar to Fig. 1
[6]. Antenna arrays, antenna feeding networks, mixed ana$\log /$ digital signals, and RF connectors are integrated in a single package substrate. IC chips and other passive components were mounted on the other side of the antenna array. Mixed analog and digital signals, such as IF band, baseband, and mmWave $\mathrm{RF}$ signals, travel through the transmission lines embedded in the module or external connectors/cables. The RF cable is one of the most critical components of the $5 \mathrm{G}$ mmWave antenna module, as the RF cable and connector carry IF, digital control, VDD, and baseband signals. Therefore, it is necessary to analyze the structure of the cable thoroughly to design the mmWave antenna module for mobile devices.

\section{A TEM MODE WAVEGUIDE FOR MOBILE 5G MMWAVE MODULE APPLICATIONS}

The first step of the RF cable design for the mobile $5 \mathrm{G}$ mmWave module is to find a proper transmission line (or waveguide) structure. $\mathrm{RF}$ cables for $5 \mathrm{G}$ mmWave modules should support a broad frequency spectrum from $\mathrm{DC}$ to $\mathrm{GHz}$ band. VDD and digital/analog control signals range from $\mathrm{DC}$ to a few hundred $\mathrm{MHz}$, and IF signals are located at $6-10 \mathrm{GHz}$. It is desirable to use a low-loss TEM (transverse electromagnetic) mode waveguide structure to minimize signal dispersion and achieve an ultra-wide operation frequency bandwidth (extremely high cutoff frequency). Furthermore, the capability of extending to a multi-signal line structure is also important for signal integrity. A multi-line circular coaxial structure satisfies most of the above conditions, but it is challenging to build a compact connector and stacked line structure. A rectangular coaxial structure is an appropriate structure to meet all the design considerations.

Fig. 2 shows the proposed structure of a rectangular coaxial waveguide. In the case of Fig. 2(a), each side of the waveguide is closed with a continuous metal wall. The structure shown in Fig.

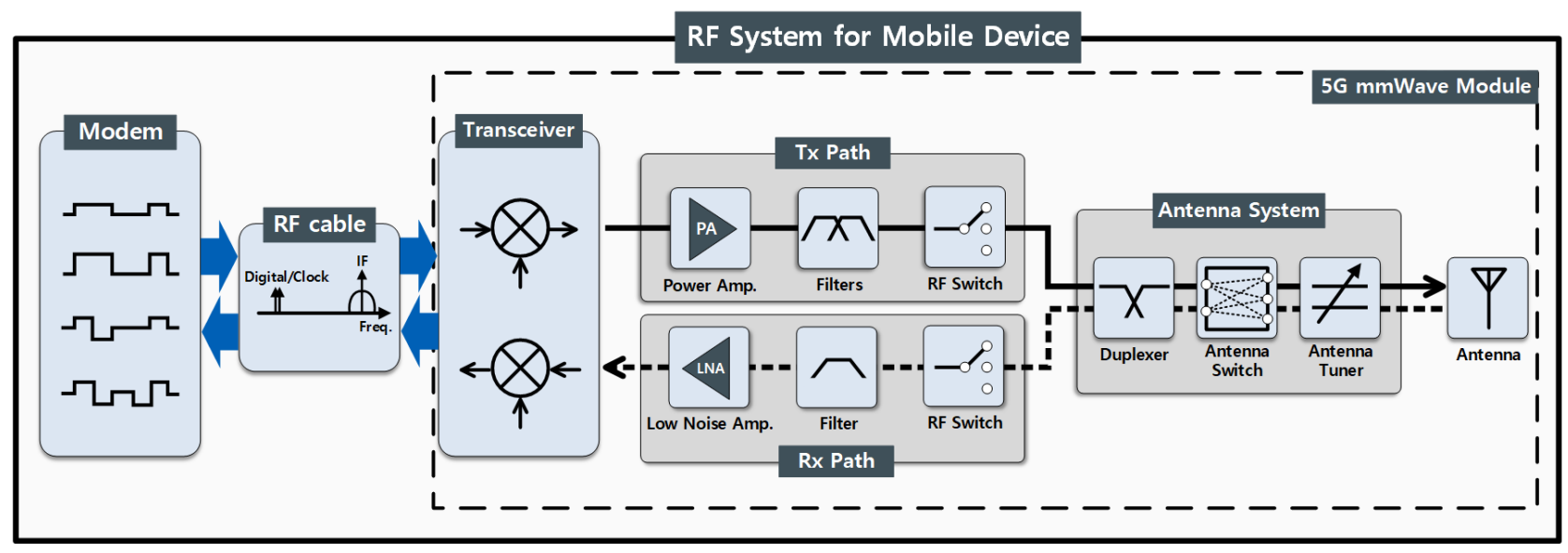

Fig. 1. The structure of a $5 \mathrm{G}$ mmWave antenna module for mobile devices. 


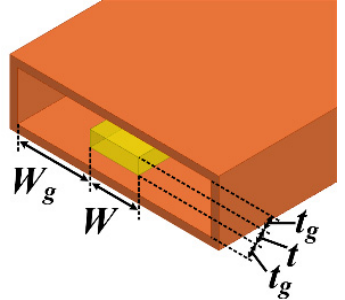

(a)

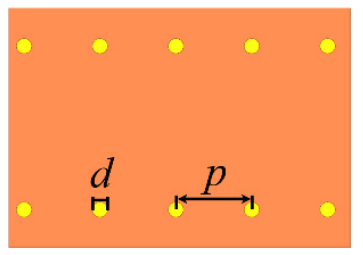

(c)

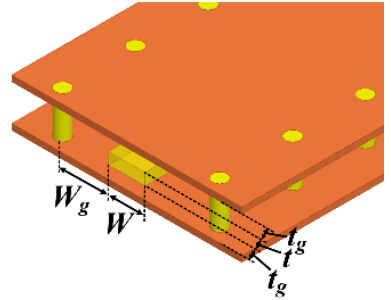

(b)

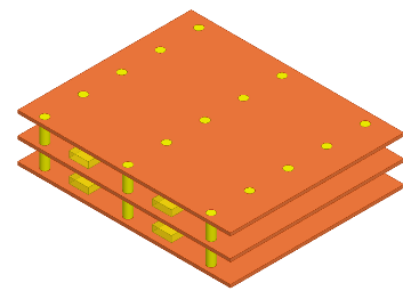

(d)
Fig. 2. (a) Rectangular coaxial waveguide with continuous side walls, (b) practical model with via walls, (c) practical model with via wall, and (d) stacked multi-line rectangular coaxial waveguide.

2(a) is a desirable structure due to complete field confinement, but it is challenging to implement a continuous metal wall on the flexible substrate. The structure using the via wall is more practical, as shown in Fig. 2(b), (c), and (d). The via pitch is a critical design parameter in this case, as it controls field leakage (radiation) from the waveguide structure and coupling between the adjacent waveguides. It is possible to achieve more than a 60-dB isolation level between the waveguides easily when each waveguide supports TEM mode waves. Fig. 2(b) and (c) show that both the left and right sides of the rectangular waveguide are closed through the via wall. It is easy and practical to extend the rectangular cable to the multi-line structure, as shown in Fig. 2(d). By sharing the via-wall or metal surface between the adjacent waveguides, the waveguides can be arranged densely without wasting space, and the electromagnetic fields can be effectively confined in the cross-section of the waveguide.

In this section, important design parameters, such as the waveguide impedance $\left(Z_{0}\right)$, loss $(\alpha)$, cutoff frequency $\left(f_{c}\right)$, and via pitch $(p)$ of the rectangular coaxial waveguide, are discussed.

\section{Impedance}

The impedance of a TEM mode waveguide is expressed as follows. $L$ and $C$ are the inductance $(L / m)$ and capacitance $(F / m)$ per unit length, respectively, $v_{p}$ is the phase velocity in the given waveguide structure and material, and $\varepsilon_{e f f}$ is the effective dielectric constant of the dielectric material. The characteristic impedance and phase velocity of a TEM mode waveguide can be expressed as Eqs. (1) and (2).

$$
Z_{0}=\sqrt{\frac{L}{C}}=\frac{1}{v_{p} C}
$$

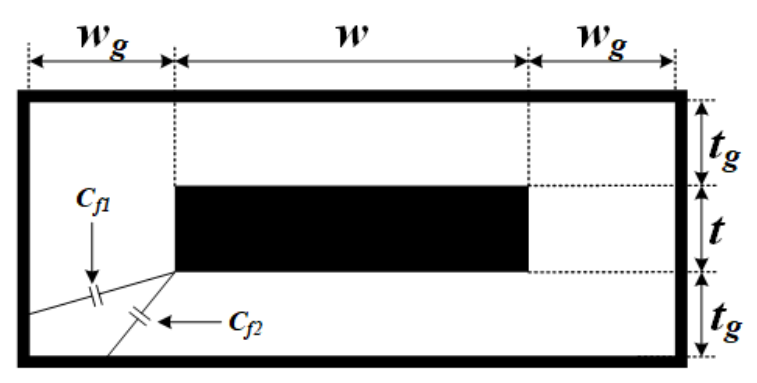

Fig. 3. Rectangular coaxial waveguide structure section and design variables.

$$
v_{p}=1 / \sqrt{\mu \cdot \varepsilon_{\text {eff }}}(\mathrm{m} / \mathrm{s})
$$

As written in (1), $C$ is a dominant parameter of the characteristic impedance value. Fig. 3 shows a cross-section of the rectangular coaxial waveguide. A rectangular signal line is located at the center of the waveguide. The impedance of the waveguide can be calculated by computing the total capacitance $\left(C_{t}\right)$ per unit length, which is obtained through the conformal mapping of the field distribution from a cylindrical to a rectangular geometry [7]. The $C_{t}$ value includes the corner capacitance values per unit length $\left(C_{f 1}\right.$ and $\left.C_{f 2}\right)$, in addition to the capacitance per unit length of the conventional circular coaxial waveguide $[8,9]$. $C_{f 1}$ and $C_{f 2}$ can be expressed as Eqs. (3) and (4), and the resulting $C_{t}$ and $Z_{0}$ are also shown in Eqs. (5) and (6).

$$
\begin{gathered}
C_{f 1}=\frac{\varepsilon_{e f f}}{\pi}\left[\log \left(\frac{w_{g}^{2}+t_{g}^{2}}{4 t_{g}^{2}}\right)+\frac{2 t_{g}}{w_{g}} \tan ^{-1}\left(\frac{w_{g}}{t_{g}}\right)\right](\mathrm{F} / \mathrm{m}) \\
C_{f 2}=\frac{\varepsilon_{e f f}}{\pi}\left[\log \left(\frac{w_{g}^{2}+t_{g}^{2}}{4 w_{g}^{2}}\right)+\frac{2 w_{g}}{t_{g}} \tan ^{-1}\left(\frac{t_{g}}{w_{g}}\right)\right](\mathrm{F} / \mathrm{m}) \\
C_{t}=2 \varepsilon_{\text {eff }}\left(\frac{w}{t_{g}}+\frac{t}{w_{g}}\right)+4\left(C_{f 1}+C_{f 2}\right) \quad(\mathrm{F} / \mathrm{m}) \\
Z_{0}=\frac{\eta_{0}}{\sqrt{\varepsilon_{e f f}}\left[2\left(\frac{w}{t_{g}}+\frac{t}{w_{g}}\right)+\frac{4}{\varepsilon_{e f f}}\left(C_{f 1}+C_{f 2}\right)\right]}(\Omega)
\end{gathered}
$$

The proposed equations were verified by comparing calculated results to the computation data of the commercial full-wave 3D simulator, HFSS v17.1 (Ansys Inc., Canonsburg, PA, USA). Fig. 4 shows the impedance variation according to the value of the signal line width $(w)$ for each rectangular coaxial waveguide in the vacuum core (Fig. 4(a)) and filled with Teflon (Fig. 4(b)). For the calculation, all the metals were set to copper $(\sigma=$ $\left.5.96 \times 10^{7} \Omega / \mathrm{m}\right)$, and the electrical properties of Teflon were defined as $\varepsilon_{r}=2.0$ and $\tan \delta=0.001$. The simulated and formulated results agree well. A designed practical $50 \Omega$ rectangular coaxial waveguide has $w_{g}=68 \mu \mathrm{m}, w=120 \mu \mathrm{m}$, $t_{g}=42 \mu \mathrm{m}$, and $t=16 \mu \mathrm{m}$.

\section{Cutoff Frequency}

A coaxial waveguide consisting of two conductors, by their nature, supports a TEM mode. The operating frequency band 


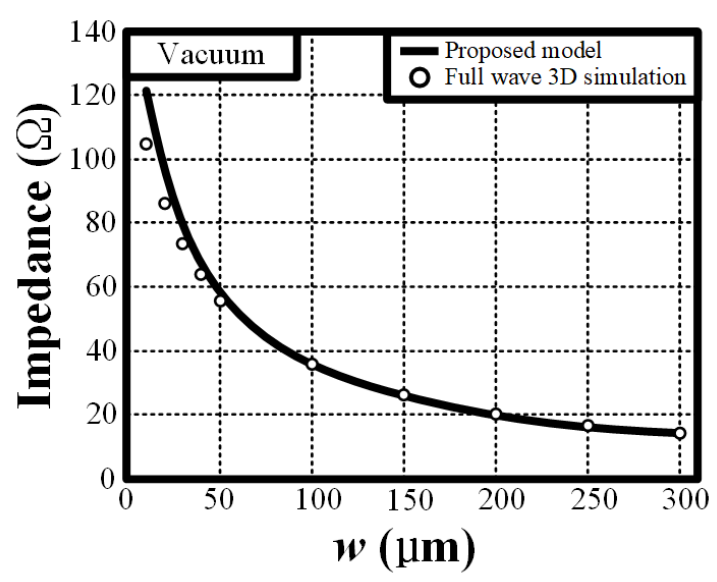

(a)

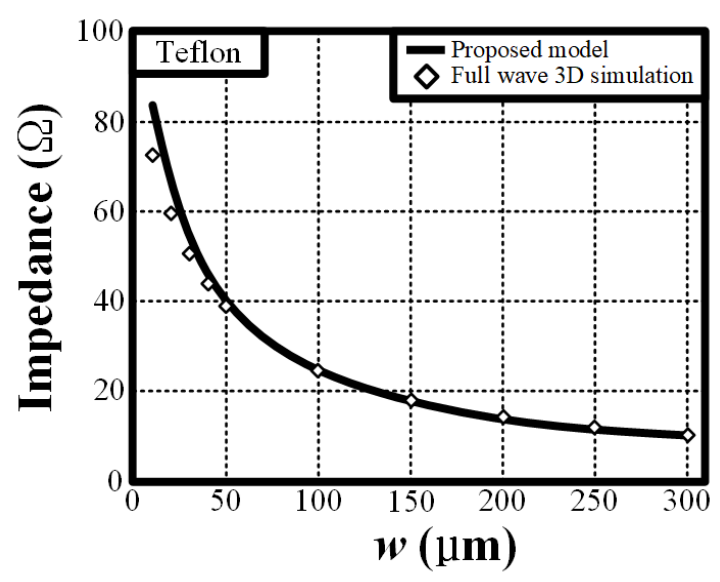

(b)

Fig. 4. Impedance change of rectangular coaxial waveguide filled by (a) vacuum and (b) Teflon.

width of a TEM mode waveguide can be defined from DC to the frequency at which any high-order wave propagation mode is excited. The first high-order mode of a square coaxial waveguide is $\mathrm{TE}_{01}$ or $\mathrm{TE}_{10}$. The cutoff frequency $\left(f_{c}\right)$ of the rectangular coaxial waveguide's $\mathrm{TE}_{10}$ mode can be expressed by the transcendental equation, and it can be calculated by Eqs. (7)-(9) [10]. For the $\mathrm{TE}_{01}$ mode, the design parameters should be modified as $t_{g} \rightarrow w_{g}, w \rightarrow t, w_{g} \rightarrow t_{g}$, and $t \rightarrow w$.

$$
\begin{gathered}
\cot \left(\frac{2 \pi t_{g} f_{c}}{v_{p}}\right)-\tan \left(\frac{t \pi f_{c}}{v_{p}}\right)=\frac{B}{Y_{C 1}} \\
\frac{B}{Y_{C 1}}=\frac{2 f_{c}}{v_{p}}\left(w+2 w_{g}\right)\left[-\ln (4 u)+\frac{1}{3} u^{2}\right. \\
\left.+\frac{1}{2}\left(1-u^{2}\right)^{4}\left(\frac{f_{c}\left(w+2 w_{g}\right)}{v_{p}}\right)^{2}\right] \\
u=\frac{2 w_{g}}{w+2 w_{g}}
\end{gathered}
$$

Because (7) and (8) are transcendental equations, it is difficult to obtain an analytical solution. In this paper, a graphical method was chosen to obtain solutions to the transcendental equa-

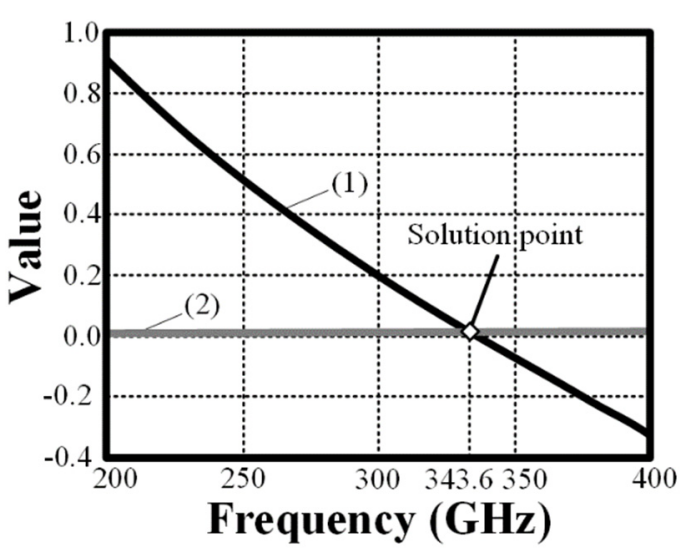

Fig. 5. Graphical analysis of high-order mode of a rectangular coaxial waveguide.

tions, as shown in Fig. 5. The values of the variables used were a $50-\Omega$ waveguide designed in section $\mathrm{A}$. The first high-order mode cutoff frequency of the $50-\Omega$ rectangular coaxial waveguide was about $334 \mathrm{GHz}$. Therefore, the operating frequency bandwidth of the designed waveguide was DC to $334 \mathrm{GHz}$, which was much higher than the operation frequency of the $5 \mathrm{G}$ mmWave module.

\section{Loss}

The total loss $\left(\alpha_{t}\right)$ of a TEM mode rectangular coaxial waveguide can be divided into three parts as, shown in (10): radiation (leakage) loss $\left(\alpha_{l}\right)$, dielectric loss $\left(\alpha_{d}\right)$, and conductor loss $\left(\alpha_{c}\right)$.

$$
\alpha_{t}=\alpha_{l}+\alpha_{c}+\alpha_{d} \cong \alpha_{c}+\alpha_{d}
$$

There is negligible radiation loss when the waveguide is closed with continuous metal walls or a fine-pitched via array. Each loss can be calculated as shown in (11) and (12) [11].

$$
\begin{gathered}
\alpha_{d}=\frac{k_{0}^{2} \tan \delta}{2 k_{z}}=\frac{k_{0} \tan \delta}{2 \sqrt{\varepsilon_{r}}} \\
\alpha_{c}=\frac{47.09 R_{S}}{\eta Z_{0}}\left(1+\frac{t+2 t_{g}}{w}\right) \frac{w}{\left[0.2794\left(t+2 t_{g}\right)+0.7206 w\right]^{2}} \\
\quad \text { for }\left(t+2 t_{g}\right) / w<2.5 \\
\alpha_{c}=\frac{59.37 R_{S}}{\eta Z_{0}}\left(1+\frac{t+2 t_{g}}{w}\right) \frac{1}{t+2 t_{g}} \\
\text { for } 2.5 \leq\left(t+2 t_{g}\right) / w \leq 4 \\
\alpha_{c}=\frac{47.09 R_{S}}{\eta Z_{0}}\left(1+\frac{t+2 t_{g}}{w}\right) \frac{1}{t+2 t_{g}} \\
\text { for }\left(t+2 t_{g}\right) / w<4
\end{gathered}
$$

$R_{s}$ is the sheet resistance of the metal $\left(R_{s}=1 /\left(\delta_{s} \cdot \sigma\right)\right), \eta$ is the intrinsic impedance $(\eta=\sqrt{\mu / \varepsilon})$ of the medium, $Z_{0}$ is the characteristic impedance of the transmission line $\left(Z_{0}=\right.$ $\sqrt{L / C})$. Fig. 6 shows the calculated and simulated attenuation constants of a rectangular coaxial waveguide made of copper and 


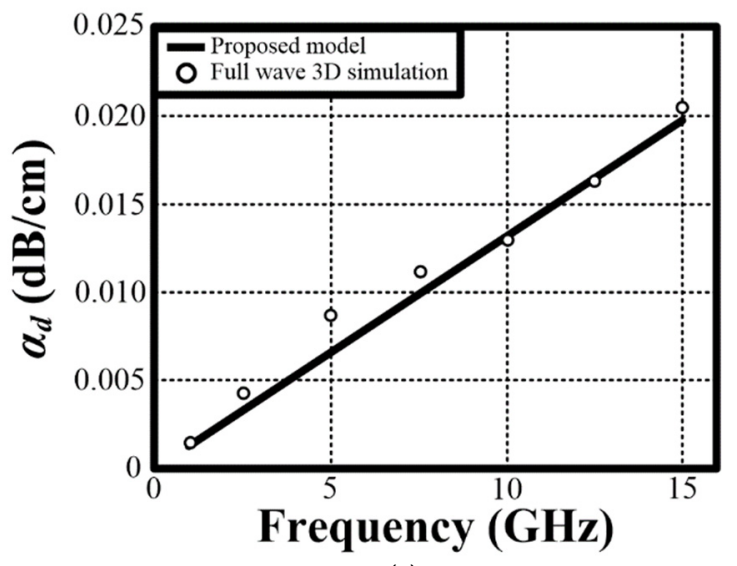

(a)

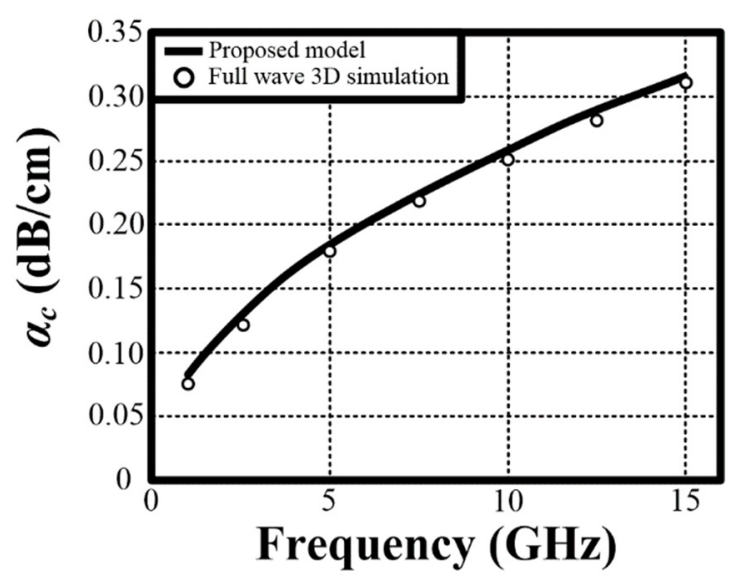

(b)

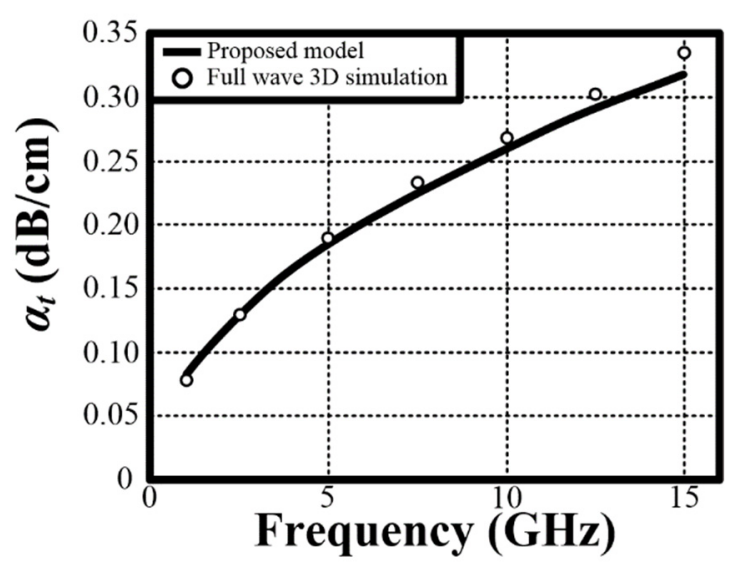

(c)

Fig. 6. Loss of rectangular coaxial waveguide composed of Teflon: (a) dielectric loss, (b) conductor loss, and (c) total loss.

Teflon. The simulated and calculated results are well matched.

\section{Via Pitch}

Continuous sidewalls can be considered as via arrays with zero pitch length but it is challenging to implement solid metal walls in the flexible RF or module substrate. The periodically arranged via wall shown in Fig. 2(b) and (c) is a more practical structure than the continuous metal wall. The critical design parameters are the diameter $(d)$ and pitch $(p)$ of the vias. The electromagnetic bandgap (EBG) should be considered because the via wall is a periodic structure $[12,13]$. From $(13)$, the first visible bandgap occurs when $n=1$.

$$
\begin{gathered}
\beta_{z}=n \pi \\
p=\frac{\pi}{\beta_{z}}=\frac{\lambda_{g}}{2}
\end{gathered}
$$

It is clear that the via pitch should be shorter than the halfwavelength, as shown in (14). The smallest via pitch is defined by mechanical durability and feasibility. It is desirable to set the minimum via-to-via distance of the fabrication process, but excessive via density decreases the mechanical durability of the designed rectangular coaxial waveguide. As a rule of thumb, the maximum via pitch should be less than a quarter wavelength of the guided wave, and the minimum via pitch is $\lambda_{g} / 20$ when the design and fabrication margins are considered. It should be noted that the maximum via pitch is a hard bound because the bandgap effect should be prevented. The minimum via pitch is a soft bound that varies depending on the manufacturing capability and process. Fig. 7 graphically presents the via design guide when the via pitch $(p)$ and diameter $(d)$ are given. The via diameter and pitch are normalized by the guided wavelength. The upper half plane is a reasonable design area, as the via diameter cannot be larger than the via pitch.

\section{Radiation}

Electromagnetic radiation is strictly regulated by law for the public health. According to the FCC regulations for electromagnetic radiation (47 CFR 15.109 Radiated emission limits) [14], mobile devices are classified as Class B, as shown in Table 1. The $5 \mathrm{G}$ mmWave module or cable should not exceed 500 $\mu \mathrm{V} / \mathrm{m}$ of electric field intensity (E) at a point 3-m away from the device under test (DUT).

Based on the given regulations and the Poynting vector theorem, the maximum allowed power leakage is obtained as follows. Because the distance $(r)$ from the DUT is $3 \mathrm{~m}$, the electromag-

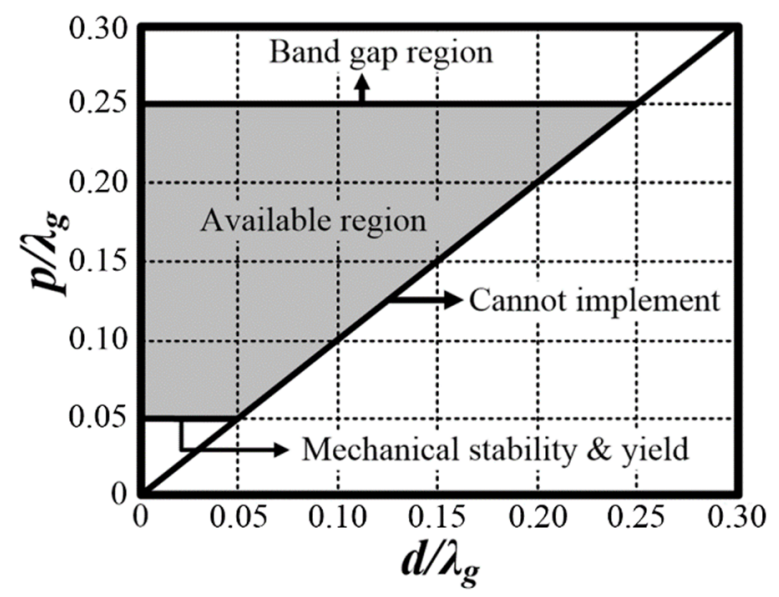

Fig. 7. Graphical analysis for the via wall design. 
Table 1. FCC regulation for Class-B devices

\begin{tabular}{cc}
\hline Frequency $(\mathrm{MHz})$ & E-field $(\mu \mathrm{V} / \mathrm{m})$ \\
\hline $30-88$ & 90 \\
$88-216$ & 150 \\
$216-960$ & 210 \\
$>960$ & 300 \\
\hline
\end{tabular}

netic field having a frequency higher than $10 \mathrm{GHz}$ can be considered the far field at the measurement point. It is reasonable to assume the radiated EM field is a TEM wave. In the spherical coordinates, the relationship between the electric field (E) and the magnetic field $(\mathrm{H})$ intensities satisfies the following conditions.

$$
\begin{gathered}
\mathbf{E}=E_{\theta} \hat{\theta} \\
\mathbf{H}=H_{\phi} \hat{\phi} \\
H_{\phi}=E_{\theta} / \eta
\end{gathered}
$$

Using Poynting's vector theorem, the total amount of radiated power $\left(P_{\text {rad }}\right)$ can be obtained as follows [15].

$$
\begin{gathered}
P_{\text {rad }}=\oiint_{S}(\mathbf{E} \times \mathbf{H}) \cdot d \mathbf{s} \\
=\oiint_{S}\left(E_{\theta} \hat{\theta} \times H_{\varnothing}^{*} \hat{\phi}\right) \cdot \hat{r} d s \\
=\oiint_{S}\left(E_{\theta} \hat{\theta} \times H_{\varnothing}^{*} \hat{\phi}\right) \cdot\left(r^{2} \sin \theta\right) \hat{r} d \phi d \theta \\
=\oiint_{S}\left(E_{\theta} \cdot \frac{E_{\theta}^{*}}{\eta_{0}}\right) \cdot(\hat{r} \cdot \hat{r})\left(r^{2} \sin \theta\right) d \phi d \theta \\
=\frac{\left|E_{\theta}\right|^{2}}{\eta_{0}} \int_{0}^{\pi} \int_{0}^{2 \pi} r^{2} \sin \theta d \phi d \theta \\
=\frac{\left|E_{\theta}\right|^{2}}{\eta_{0}} 4 \pi r^{2}
\end{gathered}
$$

where the intrinsic impedance $\left(\eta_{0}\right)$ of the free space is $120 \pi$, the radiated E-field strength, and the total radiated power are summarized as follows.

$$
\begin{gathered}
\left|E_{\theta}\right|=\sqrt{\frac{\eta_{0} P_{\text {rad }}}{4 \pi r^{2}}}=\frac{\sqrt{30 P_{\text {rad }}}}{r} \\
P_{\text {rad }}=\frac{\left(r\left|E_{\theta}\right|\right)^{2}}{30}(\mathrm{~W})
\end{gathered}
$$

Therefore, in accordance with FCC regulations, the total radiated power leakage from the designed waveguide structure should not exceed $75 \mathrm{nW}$ to generate an electric field $\left(\left|E_{\theta}\right|\right)$ less than the FCC requirement of $500 \mu \mathrm{V} / \mathrm{m}$ at a distance of 3 $\mathrm{m}$ from the DUT. It should be noted that the FCC's radiation regulation can be verified at a distance of less than $3 \mathrm{~m}$ from the DUT by evaluating (21) at any distance.

\section{CONCLUSION}

In this paper, the design and analysis of a TEM mode rectan- gular coaxial waveguide was presented. Detailed design equations for the TEM mode rectangular coaxial waveguide structure, including impedance, loss, bandwidth, vias, and radiation analysis, were also discussed. The design parameters of the $50 \Omega$ TEM mode waveguide were presented and its electrical characteristics were thoroughly studied as a design example. The proposed TEM mode waveguide design and analysis are scalable to other applications such as beyond-5G or sub- $\mathrm{THz}$ applications.

This work was supported by the National Research Foundation of Korea (NRF) grant funded by the Korea government (MSIT) (No. 2020R1C1C1003362) and the Air Force Office of Scientific Research (No. FA2386-19-1-0124).

\section{REFERENCES}

[1] A. Gupta and R. K. Jha, "A survey of 5G network: architecture and emerging technologies," IEEE Access, vol. 3, pp. 1206-1232, 2015.

[2] M. Agiwal, A. Roy, and N. Saxena, "Next generation 5G wireless networks: a comprehensive survey," IEEE Communications Surveys \& Tutorials, vol. 18, no. 3, pp. 1617-1655, 2016.

[3] Y. P. Zhang and D. Liu, "Antenna-on-chip and antenna-inpackage solutions to highly integrated millimeter-wave devices for wireless communications," IEEE Transactions on Antennas and Propagation, vol. 57, no. 10, pp. 2830-2841, 2009.

[4] S. Kim and J. Kim, "Design of reconfigurable antenna feeding network using coupled-line switch for 5G Millimeterwave communication system," Applied Computational Electromagnetics Society Journal, vol. 33, no. 8, pp. 861-867, 2018.

[5] M. Swaminathan, J. Kim, I. Novak, and J. P. Libous, "Power distribution networks for system-on-package: Status and challenges," IEEE Transactions on Advanced Packaging, vol. 27, no. 2, pp. 286-300, 2004.

[6] Qualcomm, "Qualcomm announces second generation 5G $\mathrm{RF}$ front-end solutions for sleeker, more efficient $5 \mathrm{G}$ multimode mobile devices," 2019 [Online]. Available: https:// www.qualcomm.com/news/releases/2019/02/19/qualcommannounces-second-generation-5g-rf-front-end-solutionssleeker-more.

[7] M. Lukic, S. Rondineau, Z. Popovic, and S. Filipovic, "Modeling of realistic rectangular $\mu$-coaxial lines," IEEE Transactions on Microwave Theory and Techniques, vol. 54, no. 5, pp. 2068-2076, 2006.

[8] T. S. Chen, "Determination of the capacitance, inductance, and characteristic impedance of rectangular lines," IRE Transactions on Microwave Theory and Techniques, vol. 8, no. 5, 
pp. 510-519, 1960.

[9] J. R. Reid, E. D. Marsh, and R. T. Webster, "Micromachined rectangular-coaxial transmission lines," IEEE Transactions on Microwave Theory and Techniques, vol. 54, no. 8, pp. 34333442, 2006.

[10] L. Gruner, "Higher order modes in rectangular coaxial waveguides," IEEE Transactions on Microwave Theory and Techniques, vol. 15, no. 8, pp. 483-485, 1967.

[11] K. H. Lau, "Loss calculations for rectangular coaxial lines," IEE Proceedings H-Microwaves, Antennas and Propagation, vol. 135, no. 3, pp. 207-209, 1988.

[12] D. Deslandes and K. Wu, "Accurate modeling, wave mechanisms, and design considerations of a substrate integrated

Eunyoung Park

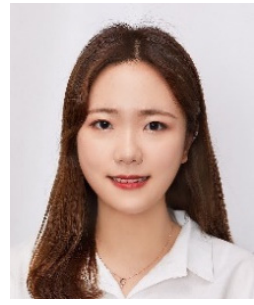

received the B.S. degree in electronics engineering at Pusan National University, Busan, South Korea, in 2020, where she is currently working toward the Ph.D. degree. Her current research interests include $\mathrm{RF}$ packaging and antenna modules for mmWave. waveguide," IEEE Transactions on Microwave Theory and Techniques, vol. 54, no. 6, pp. 2516-2526, 2006.

[13] M. Pasian, M. Bozzi, and L. Perregrini, "A formula for radiation loss in substrate integrated waveguide," IEEE Transactions on Microwave Theory and Techniques, vol. 62, no. 10, pp. 2205-2213, 2014.

[14] Code of Federal Regulations, "47 CFR 15.109 - Radiated emission limits," 2010 [Online]. Available: http//www.govinfo.gov/app/details/CFR-2010-title47-vol1/CFR-2010title47-vol1-sec15-109/summary.

[15] D. M. Pozar, Microwave Engineering, 3rd ed. Hoboken, NJ: John Wiley \& Sons, 2005.

Sangkil Kim (M'18)

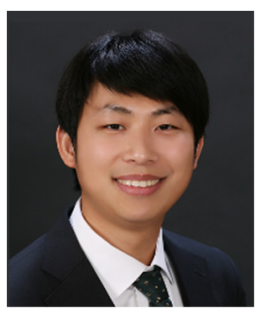

received the B.S. degree in Electrical and Electronic Engineering from Yonsei University, Seoul, South Korea, in 2010. He received his M.S. and Ph.D. degrees in Electrical and Computer Engineering from Georgia Institute of Technology, Atlanta, GA, USA, in 2012 and 2014, respectively. He visited King Abdullah University of Science and Technology, Thuwal, Saudi Arabia, in 2013; Centre Technologic Telecommunications Catalunya (CTTC), Barcelona, Spain, in 2013; and CNRS-LAAS, Toulouse, France, in 2013; as a visiting scholar. He worked on the world's first $5 \mathrm{G}$ antenna module for mobile devices at Qualcomm Inc. in 2015-2018. He is currently an Assistant Professor in the Department of Electronics Engineering, Pusan National University, Busan, South Korea. His work has been covered in 25 peer-reviewed journals, 38 international conference proceedings, and five book chapters. Dr. Kim was the recipient of the 2015 IET Microwaves, Antennas and Propagation Premium Award and the 2018 Pusan National University Department of Electronics Engineering Distinguished Faculty Achievement Award. He is a committee member of MTT-24 RFID Technologies since April 2018. 